\title{
Fully distributed mobility management scheme for future heterogeneous wireless networks
}

\begin{abstract}
Mobile network operators urgently need to scalable and reliable mobility management solutions to cope with the explosive increase of the mobile users and internet traffic. Although the network-based PMIPv6 protocol is considered the favorable solution to solve the problems of host-based MIP protocol, these protocols are based on centralized mobility management CMM scheme. Current mobility solutions posing several challenges due to heavily centralized architecture. Furthermore, the future 5G networks are based on flat infrastructures to reduce the load in the network core. The distributed mobility management DMM scheme is introduced recently to overcome the problems of CMM. Additionally, to maintain the heterogeneity of future wireless networks, IEEE 802.21 Media Independent Handover (MIH) framework identifies the structure and services to provide seamless handover in heterogeneous networks. In this paper, we develop an efficient network-based fully DMM scheme based on the cross layer design of layer $2 \mathrm{MIH}$ and layer 3 PMIPv6 protocols. The proposed approach removes any central anchor node in the network infrastructure and also eliminates any layer 2 and layer 3 signaling between the mobile node and the access networks. The numerical evaluation shows that the efficient approach gives enhance handover performance in terms of signaling cost, handover latency, and packet loss.
\end{abstract}

Keyword: Cross layer design; Distributed mobility management; Handover; MIH; PMIPv6 